\title{
Digital Image Compression using Karhunen-Loève Transform
}

\section{Ghada Thanoon Younes}

\author{
College of Computer Science and Mathematics
}

University of Mosul, Mosul, Iraq

Received on: 06/02/2012

Accepted on: 19/04/2012

\begin{abstract}
In this research present the digital image compression using by Karhunen-Loève Transform (KLT), by convert a color digital image to a gray square digital image, then select the no. of eigen values and eigen vectors that can reconstruct the image, that be very near to the original image.

And then calculate compression ratio and a high result reach it, after applied fidelity criteria on image produce from compression represented by (PSNR, MSE, correlation coefficient and compression ratio), and using a matlab language programming for execute this research.
\end{abstract}

Keywords: digital image, compression, Karhunen-Loève Transform,

$$
\begin{aligned}
& \text { كبس الصور الرقمية باستخلام تحويل كارنوف لوف } \\
& \text { غادة ذنون يونس } \\
& \text { كلية علوم الحاسوب والرياضيات } \\
& \text { جامعة الموصل، الموصل، العراق } \\
& \text { تاريخ قبول البحث: 2012/04/19 } \\
& \text { تاريخ استلام البحث: 2012/02/06 }
\end{aligned}
$$

\section{الملخص}


الاستخدام الأمثل تكمـن بالإمكانيات والمـوارد المتوفرة بتقليل الكلفة مـن جهـة ممـا يـؤدي إلى تقليل وقت النقل

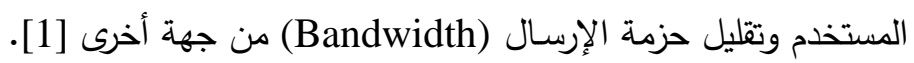

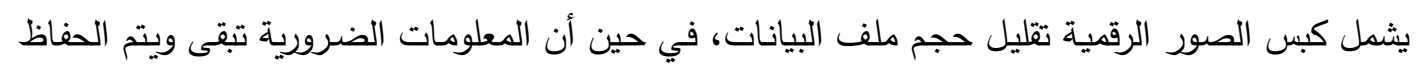
عليها, يطلق على الملف الذي تم تقليصه ملف الكبس وهو الذي يتم اعتماده في بناء الصورة الأصلية. إن المفتاح لهيكل الكبس الناجح يأتي مع التعريف الدقيق للمعلومات الضرورية، ولفهم هذا يجب التمييز بين البيانات ( data )

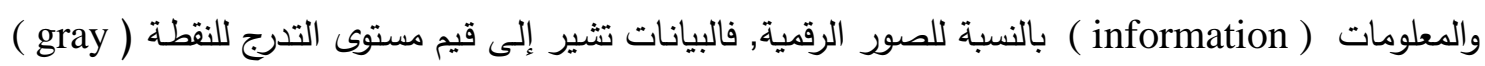

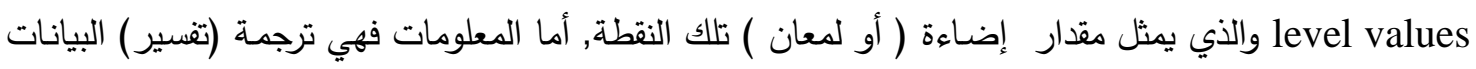

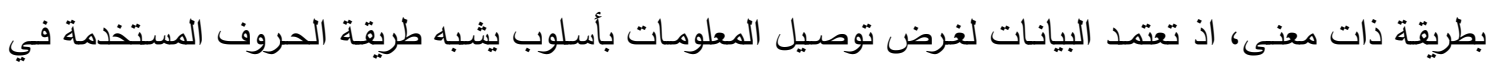

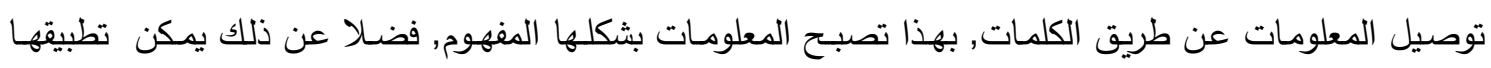
بشكل محدد,على سبيـل المثال في الصورة الثنائيـة ( binary image) التي تحتوي على نص نص المعلومات الضرورية فقط أما أن تثمل النص المراد قراءته فحسب. أو في الصور الطبية فالمعلومات الضرورية ربما تثمل التفاصيل الدقيقة في الصورة الأصلية كلها[1]]. إن التمثيل الرقمي (coding) للصورة يتطلب عددا كبيرا جدا من القيم. ومن المهم في كثير من التطبيقات أن يتم التفكير في تقنيات لتثثيل الصورة أو المعلومات. وبالرغم من أن الهدف من الترميز هو تخفيض التهن المعطيات

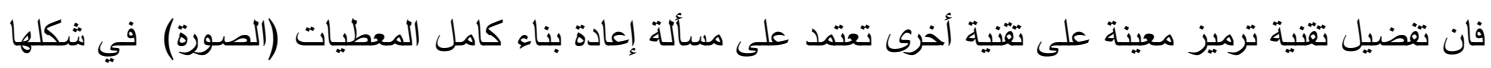
الرمزي.

ونجاح أية تقنية ترميز يعتمد على درجة ملاءمتها لبنية هيكل المعطيات, وإن الطريقة المثالية في تصميم

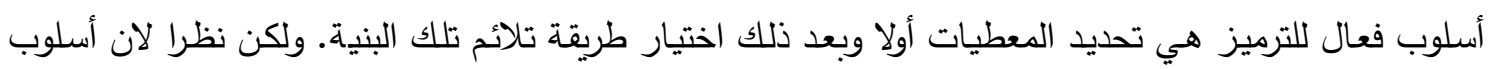
الترميز للصورة غالبا ما يتضمن قدرا من التجريب (أي بالاعتماد على المحاولة والخطأ), فالاعتبار الأكثر أهمية

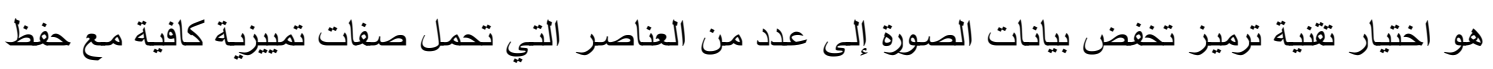
كافي للمعلومات[2].

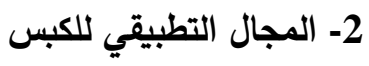

كبس الصورة مهم جدا ويطبق بمجالات عديدة في حياتتا منها :- في مجال التراسل بمختلف أنواعها, والوثائق, والنقل,شبكة الاتصالات عبر الإنترنت. كذلك طبق بثكل واسع في مجال الوسائط المتعددة, وفي مجال

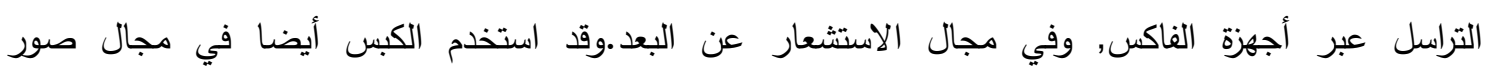
الرسومات[7].

اعتمد كثير من النماذج والأساليب على كبس الملفات, وقد جرت بثكل واسع عمليات الكبس على أسلوبين هما(المادي والبرمجي): •امتاز الأسلوب الأول بسرعة التطبيق وسهولة الاستخدام ويطبق بشكل واسع عند الحاجة إلى كبس المعلومات واكتسابها. • أما الأسلوب الثاني فقد اعتمد على الإمكانيات البرمجية لأجهزة الحاسبات وقد تمكن كثير من الباحثين من بناء خوارزميات لذلك الغرض, إذ أن خوارزميات الكبس طورت بواسطة اخذ محاسن التكرار والموجودة في بيانات 


$$
\begin{aligned}
& \text { وهناك ثلاثة أنواع رئيسية من التكرار ممكن أن تحتويها الصورة:- }
\end{aligned}
$$

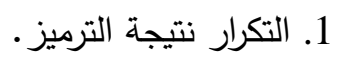

$$
\begin{aligned}
& \text { 2. النقاط المتداخلة. } \\
& \text { 3. التكرار. } \\
& \text { 3- 3قنيات كبس الصور }
\end{aligned}
$$

تصنف خوارزميات الكبس الى عدة طرائق, اذ يعتمد الكبس على أنواع البيانات المراد كبسها وحسب تركيبها والخواص التي تحتويها تلك البيانات, إذ أن هنالك بعض الخوارزميات تعمل بثكل جيد على بعض الأصناف من

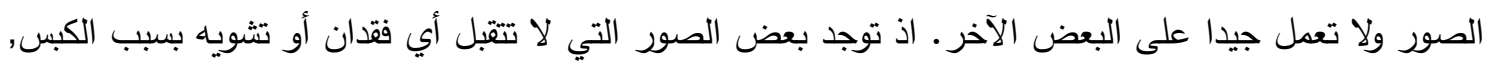

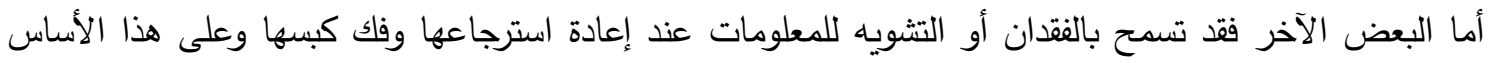

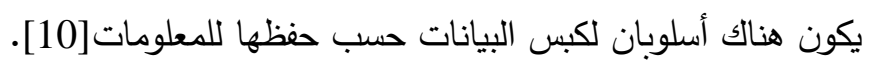

(compression lossless) الكبس بدون فقدان

في هذا النوع من الكبس من الممكن إعادة البيانات التي تم كبسها بثكل يطابق البيانات الأصلية, وتم

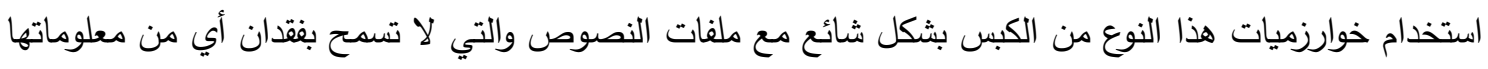
أثناء عملية الكبس, وكذلك استخدم مع ملفات الصور الطبية, والصور الفضائية, وأرشفة الصور والوثائق, والأعمال

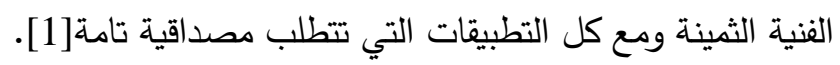

$$
\begin{aligned}
& \text { ومن طرائق الكبس بهذا الأسلوب: } \\
& \text { • طريقة هوف مان } \\
& \text { • طريقة الترميز الرياضي }
\end{aligned}
$$

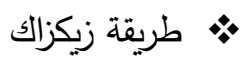

(lossy compression) الكبس بفقدان) 2-3

ومن أهم مميزات هذا الأسلوب هو الحصول على نسبة كبس عالية ولكن على حساب فقدان جزء من المعلومات الأصلية وعليه يكون استخدامه من عدمه معتمدا على نسبة فقدان البيانات المسموح بها وأهميتها. ولكن فئن

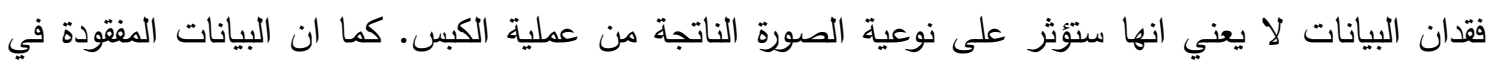

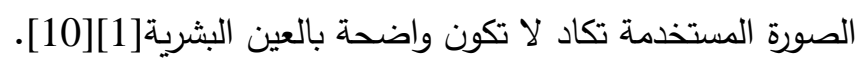

$$
\begin{aligned}
& \text { ومن طرائق الكبس بهذا الأسلوب: }
\end{aligned}
$$

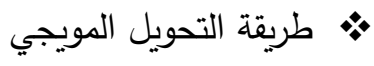

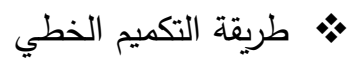

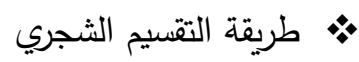

$$
\begin{aligned}
& \text { • طريقة التحويل الرمزي }
\end{aligned}
$$


4ransform coding ترميز البيانات

هي طريقة لترميز البيانات المستخدمة في مجالات كبس الصور الرقمية, يتم تحويل البيانات الى فضاء اخر قبل عملية الترميز • والفائدة من هذا هو لتحويل البيانات المدخلة الى صيغة مختلفة مما يجعل اجراء عملية الكبس لهبل اسهل وافضل، ومن المكن تجاهل بعض المعلومات التي لا تودي الى خسارة معلومات في الصورة الاصلية[10].

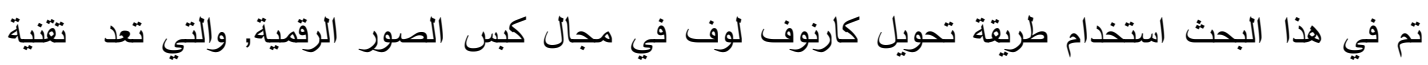

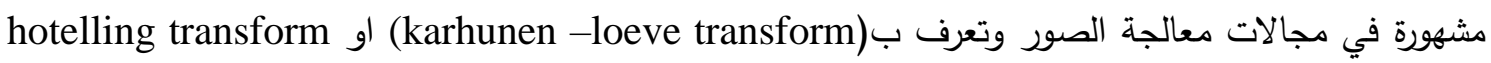
او eigenvector transform كل هذه التسميات مرتبطة وقريبة جدا من component analysisprinciple الذي يستخدم بثكل واسع في تحليل البيانات واستخلاص الخواص. اذ ان هذا التحويل يعتمد عل خصائص احصائية لنقاط الصورة او مواصفات وصفات الاثكال داخل الصورة [4]. ان طريقة كارنوف لوف استخدت في عدة حقول خاصة في مجال الاحصاء, الاتصالات, الرؤية الحاسوبية وفي مجالات ومهام عدة خاصة في تمييز الانماط ومنها تمييز الوجه،تمييز الاشكال، تخمين مسار الحركة، تعليم المتقدم وتتبع الاشكال [5] وكما تعد طريقة مثالية لتقريب مجموعة من المتجهات او الصور والتي تستخدم في مجال معالجة الصور والرؤية الحاسوبية لعديد من المهام وان العمليات الحسابية تكون بطبيعتها محددة في هذا التطبيق [6]. ان هذه الطريقة تجعل المعلومات التي لها اهمية اكبر بالصورة ظاهرة وواضحة, سيكون هذا التحويل اكثر

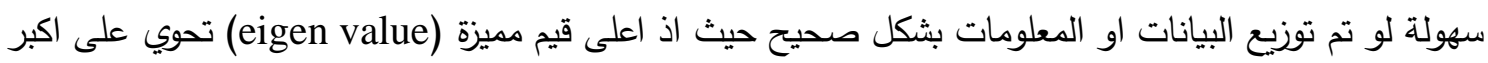
نسبة من المعلومات [9]. تعد طريقة كارنوف لوف الأمثل وذلك: تستطيع ارجاع البيانات الاصلية بالاعتماد على عمليات احصائية مستقلة . من المكن استخدام اقل عدد من معاملات التحويل والتي تحوي اكبر نسبة من البيانات. ونظرا لهذه الميزات تم استخدام كارنوف لوف ولكن هناك بعض السلبيات اذ انها تحتاج الى عمليات حسابية عديدة من اجل اجراء عملية التحويل[8]. 5- خوارزمية تحويل كارنوف لوف:

ان الخطوات التي توضح خوارزمية كارنوف لوف تم ادراجها بالنقاط الاتية:

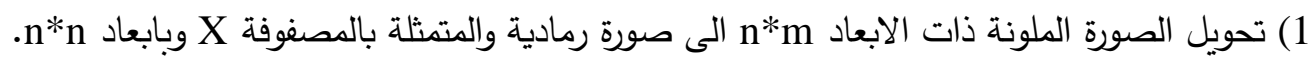

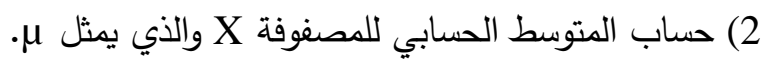

$\mu=\frac{1}{N^{*} N} \sum_{i=1}^{N} \sum_{J=1}^{N} X_{[i][j]} \quad i=1 . . N \quad, \quad J=1 \ldots . N$

3) اجراء عملية طرح المتوسط الحسابي من المصفوفة X عن طريق المعادلة الاتية:

$Z_{[i][j]}=X_{[i][j]} \quad-\mu \quad i=1 \ldots \ldots . N \quad, \quad j=1 \ldots . . N$

4) تكوين مصفوفة التباين للمصفوفة z والتي تمثل بـ C عن طريق المعادلة:

$C_{i, j}=\sum_{i=1}^{N} \sum_{j=1}^{N} \frac{Z_{[i][j]} \times Z_{[i][j]}}{N * N} \quad i=1 \ldots N, j=1 \ldots . N$ 


$$
\text { والتي سينتج عنها المصفوفة الاتية: }
$$

5) حساب القيم المميزة والمتجهات المميزة (Eigen value,Eigen vector) لمصفوفة التباين C عن طريق n*n استخدام الدالة الجاهزة بلغة ماتلاب (eig) والتي سينتج عنها مصفوفة من القيم المميزة وبابعاد التهنيز

$$
\text { 6) ومصفوفة ثانية من المتجهات المميزة وبابعاد الختيار متجهات الخواص او الصفات: }
$$

تتميز طريقة كارنوف لوف بان المتجهات المميزة التي تحمل قيمة اكبر ستحوي على نسبة اكبر من المعلومات او الخواص داخل الصورة ،وبذلك يمكن اختزال او تجاهل بعض هذه القيم التي لا تحمل نسبة عالية من البيانات اذ ترتبط القيم المميزة طرديا مع كمية البيانات التي تمثلها. لذلك سيتم ترتيب مصفوفة القيم المميزة تنازليا من الاكبر الى الاصغر وعلى اساس هذا الترتيب سيتم اعتماد ترتيب مصفوفة المتجهات المميزة (كمثال لو ان السطر الرابع من القيم المميزة يحمل القيم الاكبر

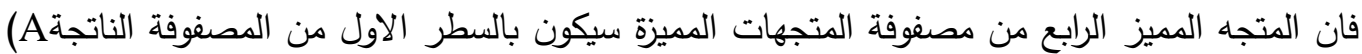
$\mathrm{A}=\left(\mathrm{v} 1, \mathrm{v} 2, \mathrm{v} 3 \ldots \ldots \ldots \ldots . . . \mathrm{v}_{\mathrm{n}}\right)$ لذلك سيكون لدينا المصفوفة

والتي ستحوي المتجهات المميزة فقط وستكون ابعاد المصفوفة A هي n*n . 7) تكوين مجموعة من البيانات الجديدة من خلال تطبيق المعادلة :

Final data $=A^{*}(X-\mu)$

8) استرجاع الصورة الاصلية من اجل ارجاع الصورة الاصلية سيكون بتطبيق المعادلة الاتية :

Data adjust $=\mathrm{A}^{\mathrm{T}} *$ Final data

$\mathrm{X}=\mathrm{AT}^{*}($ Final data $+\mu)$

هذه المعادلة يتم تطبيقها عندما لا يتم استخدام كل المتجهات المميزة في عملية استرجاع الصورة (وهذا ما يعرف باستخلاص الخواص) حيث سيساعد ( ب المتوسط الحسابي) في جعل عملية استرجاع البيانات

الاصلية صحيحة][4].

6- التطبيق العملي لخوارزمية (KLT): الخطوات الاتية توضح التطبيق العملي للطريقة المقترحة والتي تكون كالاتي:-

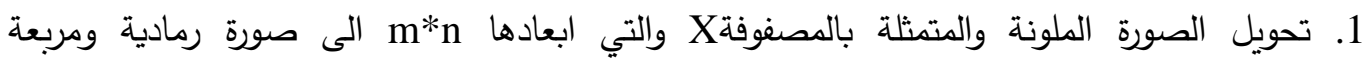
بأبعاد n*n وحسب المثال الاتي فان ابعاد الصورة هي (252*252). 


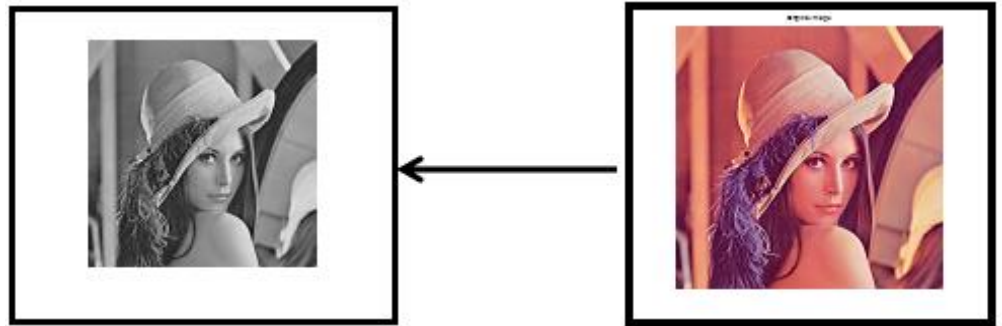

شكل (1) تحويل الصورة الاصلية الى صورة رمادية

2. تطبيق خوارزمية KLT على المصفوفة X الموضحة في الخطوة 5 من الخوارزمية (KLT).

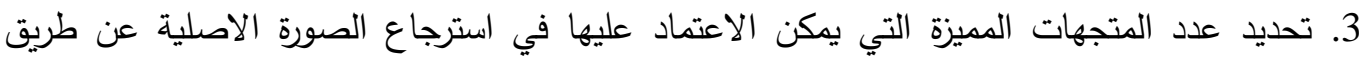

تطبيق المعادلة (6).

4. وفيما يلي نتائج تطبيق الخوارزمية:

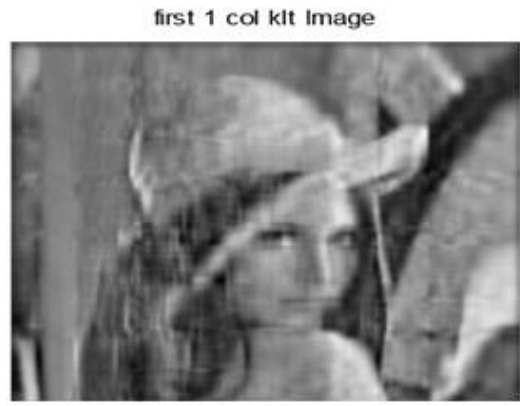

شكل(2) استرجاع الصورة من متجه مميز واحد

first $20 \mathrm{col}$ klt Image

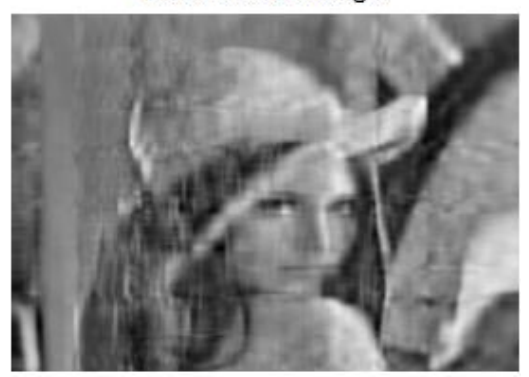

شكل(3) استرجاع الصورة من 20 متجه مميز

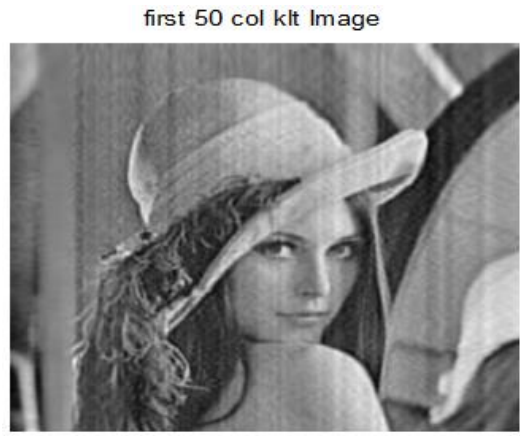

شكل(4) استرجاع الصورة من 50 متجه مميز 


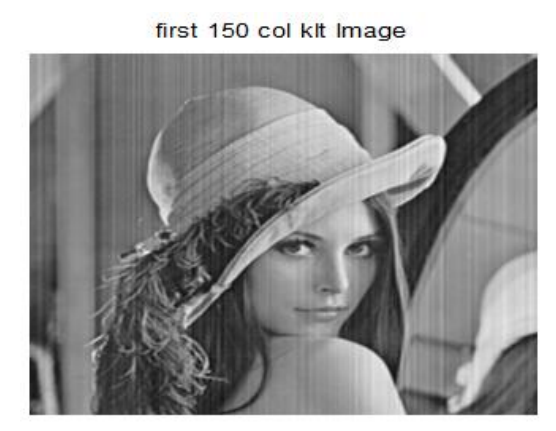

شكل (5) استرجاع الصورة من 150 متجه مميز

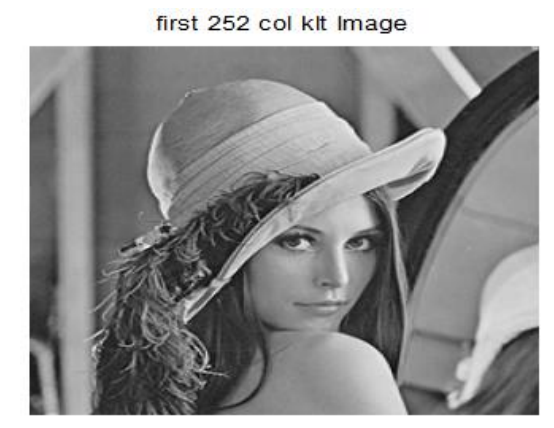

شكل(6) استرجاع الصورة من 252 متجه مميز

نلاحظ من الثكل رقم (2) انه بالاعتماد على اول متجه مميز من المصفوفة A والذي ابعاده (252*1) تم

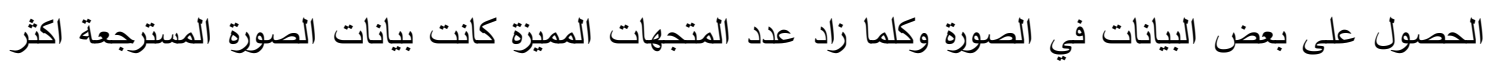
واوضح المعالم • وكما موضح في الثكل (6) الذي تم فيه استرجاع البيانات كاملة وهي اقرب بكثير من الصورة

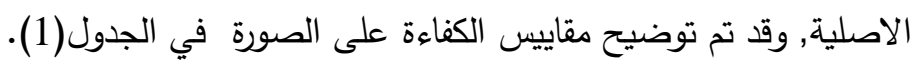
وان كمية البيانات او المعلومات الخاصة بالصورة باستخدام تحويل كارنوف لوف موجودة في الربع الاول من المتجه المميز , اما البقية فتحوي كمية قليلة من البيانات وكما موضح بالثمات بالثكل الاتي first 200: 252 col klt Image

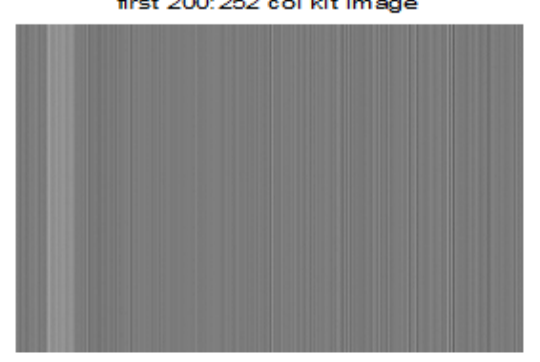

شكل(7) استرجاع الصورة من المتجه المميز رقم 200 الى 252

ونلاحظ من الثكل رقم (7) انه بالاعتماد على قيم المتجه المميز في المصفوفة A من 200 الى 252 ان

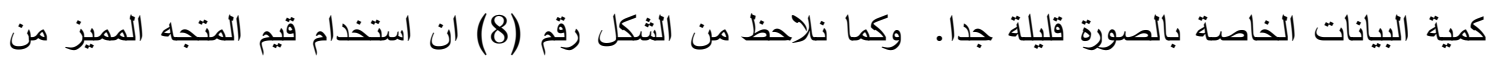
100 الى 252 اعطت ايضا نسبة قليلة من بيانات الصورة. 


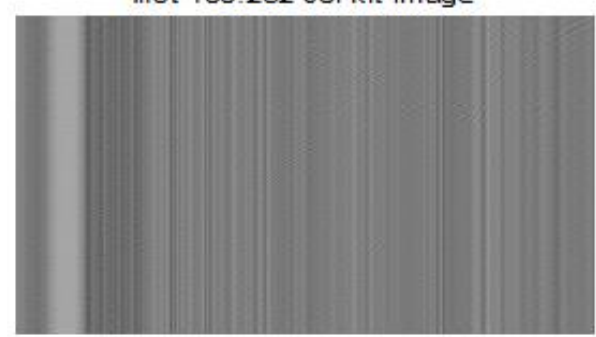

شكل(8) استرجاع الصورة من المتجه المميز رقم 100 الى 252

من هنا تم التوصل الى فكرة استخدام كارنوف لوف في مجال كبس الصور الرقمية وذلك لان اول جزء من المتجه المميز اعطى كمية كبيرة من بيانات الصورة ويمكن الغاء بقية قيم المتجه المميز وذلك لاحتوائه على كمية قليلة من البيانات والاستفادة منه في مجال الكبس لذا فان طريقة كارنوف لوف بالإمكان ادراجها ضمن طرائق الكبس بفقدان.

\section{7idelity criteria : مقاييس الكفاءة}

تم اقتباس مقياس الكفاءة من مجالات معالجة الإشارات الرقمية و نظرية المعلومات اذ تم استخدامها

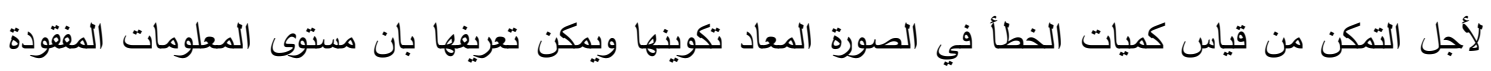

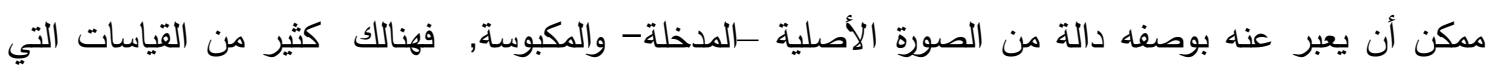

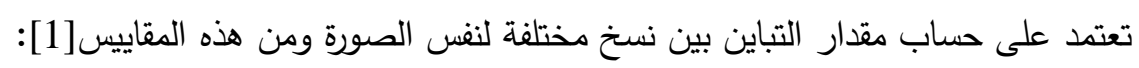
أ- نسبة مربع الخطأ Mean Square Error

$\operatorname{MSE}=\frac{1}{n * m} \sum_{i=1}^{n} \sum_{j=1}^{m} e r_{i j}^{2}$

$$
\text { حيث ان : }
$$

m,n

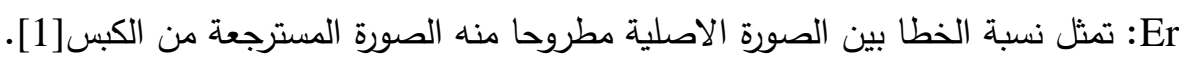

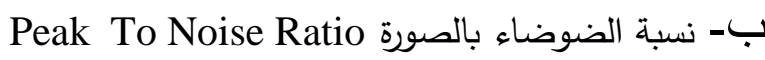
حساب PSNR باستخدام المعادلة

$$
P S N R=10 \log _{10}\left[\frac{R^{2}}{M S E}\right]
$$

$$
\text { حيث ان: }
$$

R اعلى قيمة للتدرجات الرمادية في بيانات الصورة وغالبا تساوي قيمة 255 في حالة كون الصورة المستخدمة

$$
\text { رمادية (gray image) }
$$

\section{تـ- نسبة الكبس Compression Ratio}

يمكن حساب النسبة الأصلية للكبس من خلال حجم الصورة الأصلية الرمادية غير المكبوسة وحجم

$$
\begin{aligned}
& \text { الصورة الرمادية المكبوسة وهذه النسبة للكبس ممكن التعبير عنها كما يلي[1]: } \\
& \text { compression ratio }=\frac{\text { compress image size }}{\text { uncompress image size }}
\end{aligned}
$$


Compress image size Uncompress image size

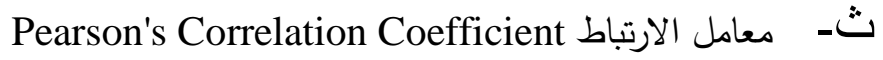
يعد من اكثر المقاييس شيوعا والمعتمدة على اجراء عملية تقسيم قيمة التباين لمتغيرين على الانحراف $P x y=\frac{\operatorname{cov}(X, Y)}{\sigma X \sigma Y}=\frac{E(X-\mu x)(Y-\mu y)}{\sigma X \sigma Y}$ حيث ان: X Y الصورة المسترجعة . Cov(x,y) هXoY لذا من المككن استخدام معامل الارتباط في حساب مدى تطابق الصورة الاصلية والمسترجعة من عملية الكبس]11].

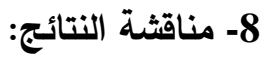
تم في هذا البحث استخدام طريقة كارنوف لوف لكبس الصور الرقمية واجريت الاختبارات على العديد من

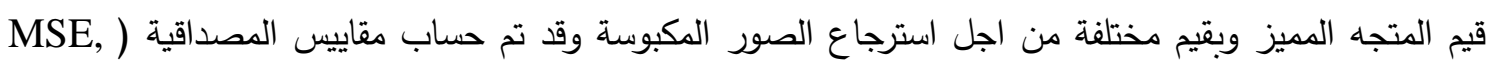
PSNR, correlation, compression rate لكل الصور المسترجعة وقد اعطت جميعها نتائج جيدة وكما موضح بالجدول(1), علما ان الصورة التي طبقت عليها الخوارزمية هي لصورة ذات التدرج الرمادي وابعاد الصورة

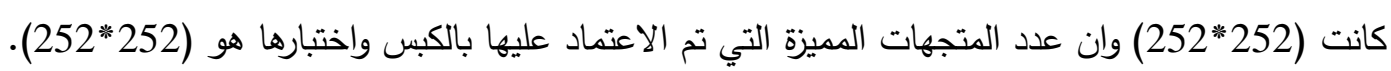
جدول(1) نتائج تطبيق مقاييس الكفاءة للصور المعتمدة على متجهات مميزة مختلفة

\begin{tabular}{|c|c|c|c|c|c|c|}
\hline $\begin{array}{c}\text { No. of } \\
\text { eigenvectors }(R, C)\end{array}$ & MSE & $\begin{array}{l}\text { Correlation } \\
\text { Coefficient }\end{array}$ & PSNR & $\begin{array}{c}\text { Compression } \\
\text { Ratio }\end{array}$ & $\begin{array}{c}\text { Size of image } \\
\text { before } \\
\text { compression }\end{array}$ & $\begin{array}{c}\text { Size of matrix } \\
\text { after } \\
\text { compression }\end{array}$ \\
\hline$(1,1: 252)$ & 0.0280 & 0.4550 & 15. 1789 & 0.0040 & 63504 & 252 \\
\hline$(1: 10,1: 252)$ & 0.0091 & 0.8681 & 20.0769 & 0.0397 & 63504 & 2520 \\
\hline$(1: 50,1: 252)$ & 0.0037 & 0.9478 & 23.963 & 0.1984 & 63504 & 12600 \\
\hline$(1: 100,1: 252)$ & 0.0024 & 0.9661 & 25.7801 & 0.3968 & 63504 & 25200 \\
\hline$(1: 150,1: 252)$ & 0.0019 & 0.9736 & 26.8419 & 0.5952 & 63504 & 37800 \\
\hline (1: 200, 1: 252) & $9.7573 \mathrm{e}-004$ & 0.9864 & 29.7592 & 0.7937 & 63504 & 50400 \\
\hline$(1: 252,1: 252)$ & 3. $0519 \mathrm{e}-031$ & 1 & 55. 8068 & 1 & 63504 & 63504 \\
\hline
\end{tabular}

نلاحظ من الثكل (9) انـه كلما زادت عدد قيم المتجهات الميزة زاد معامل الارتباط حتى وصل للواحد 1 المد بمعنى ان الصورة المسترجعة من الكبس هي جدا قريبة للصورة الاصلية.

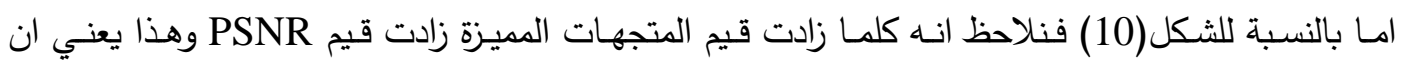
الصورة المسترجعة قريبة من الصورة الاصلية. اما بالنسبة للشكل(11) فنلاحظ انه كلما قلت قيم المتجهات المميزة كانت نسبة الكبس افضل. 
والثكل(12) نلاحظ انـه كلمـا زادت قيم المتجهات المميزة كانت قيمـة MSE قليلـة وهذا يعني ان نسبة الخطأ بالصورة قليلة وتكون الصورة المسترجعة اقرب للصورة الاصلية.

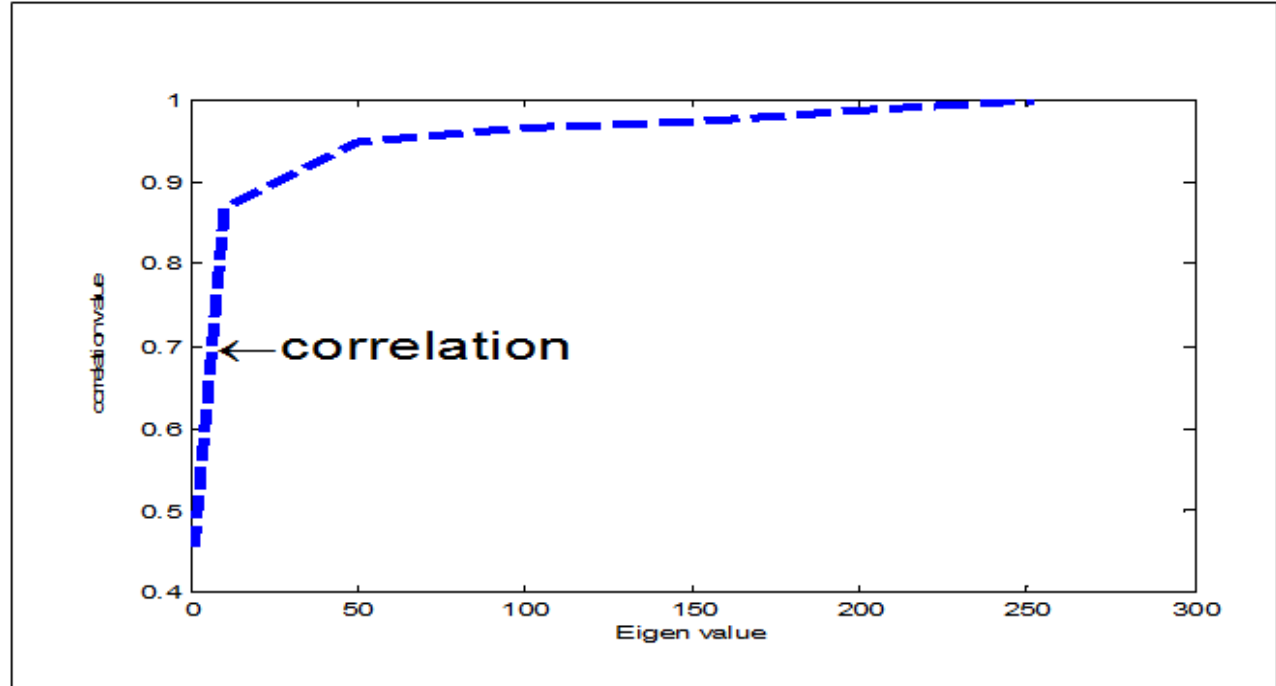

شكل (9) العلاقة بين معامل الارتباط وعدد المتجهات المميزة

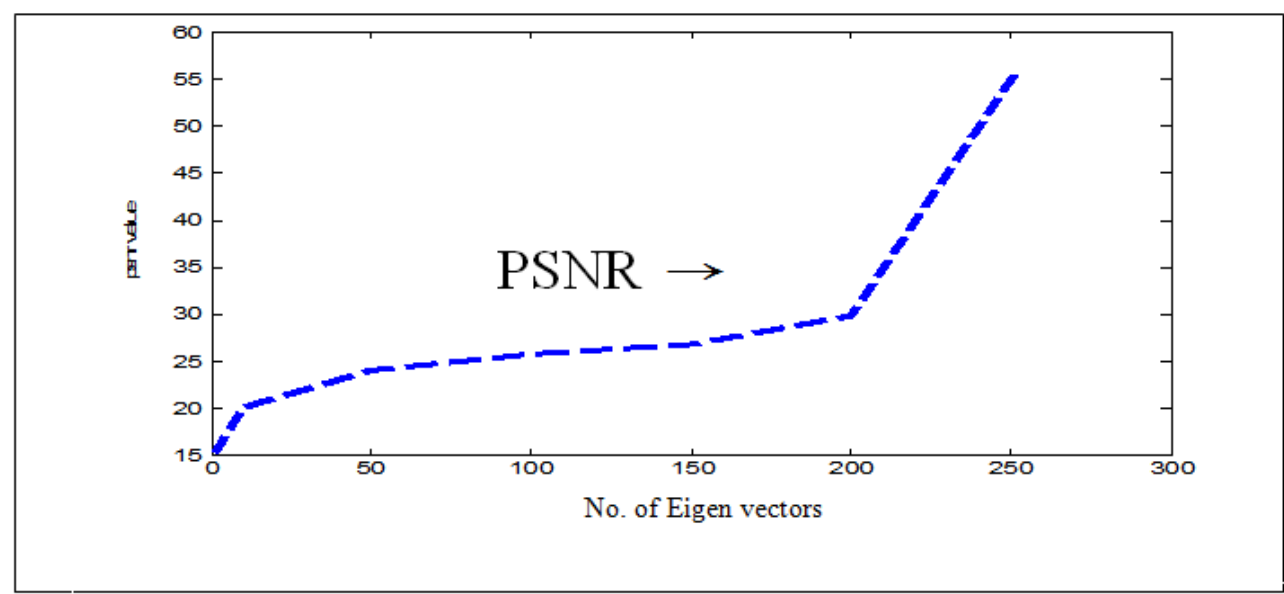

شكل(10) العلاقة بين المتجه المميز وقيم PSNR

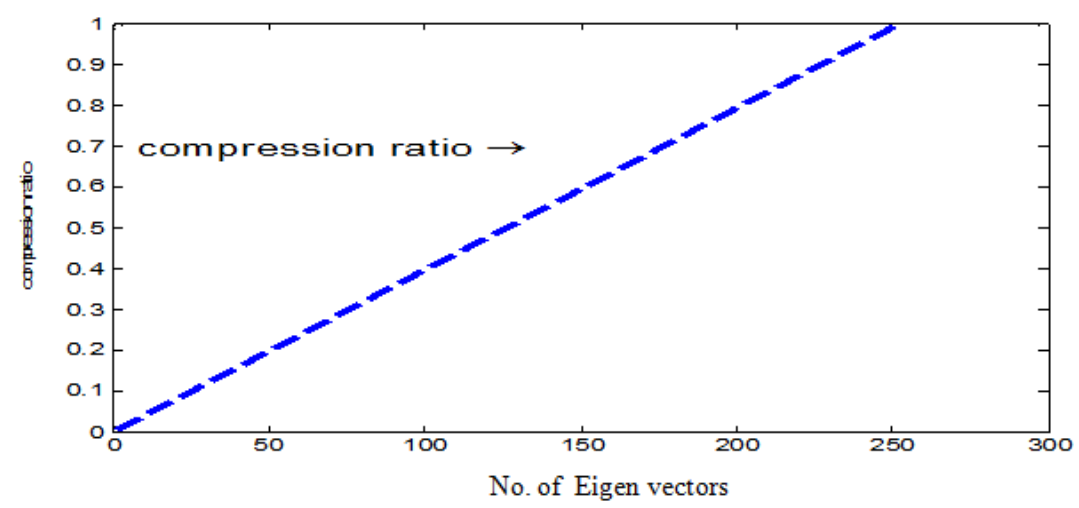

شكل(11) العلاقة بين نسبة الكبس وقيم المتجه المميز 


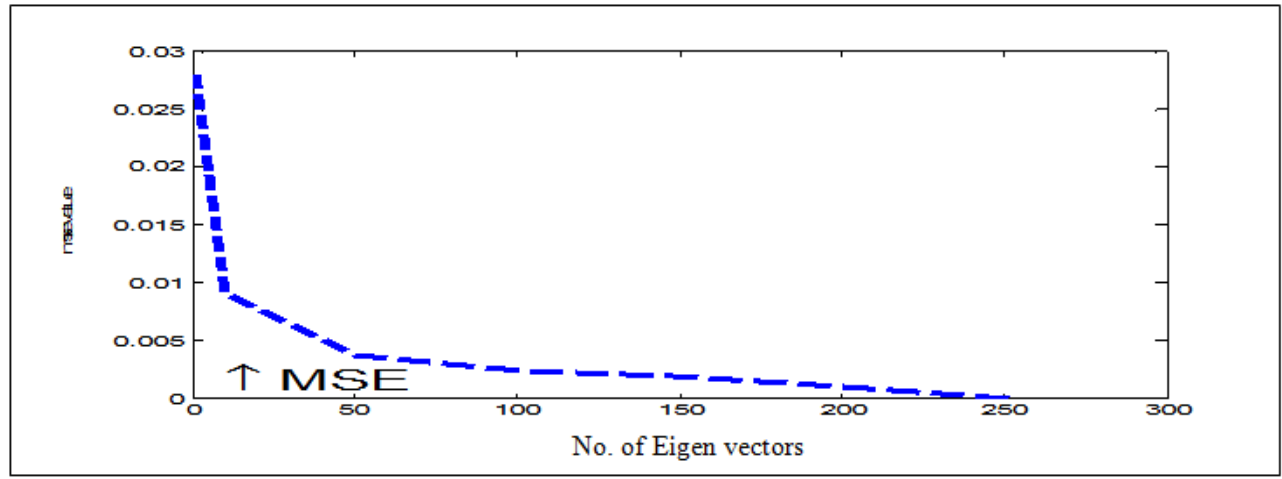

شكل(12) العلاقة بين MSE وقيم المتجه المميز

9- مقارنة مع تطبيقات اخرى

تم استخدام احد الطرائق المعروفة والمشهورة في مجال كبس الصور الرقمية وهي التحويل المويجي :wavelet

(EZW Embedded Zero tree Wavelet) 区 وهي احد طرائق التحويل المويجي المعروفة والتي تعتمد على تقسيم الصورة الى اربعة مستويات ويكون التقسيم بالنسبة للصورة على الثدة ذات القيم الاعلى ويستمر التقسيم الى ان نصل لقيمة العتبة المثبتة ويتركز التقسيم على الربع الاول لان تركيز المعلومات يكون اكبر [11] وكما موضح بالثكل(11).

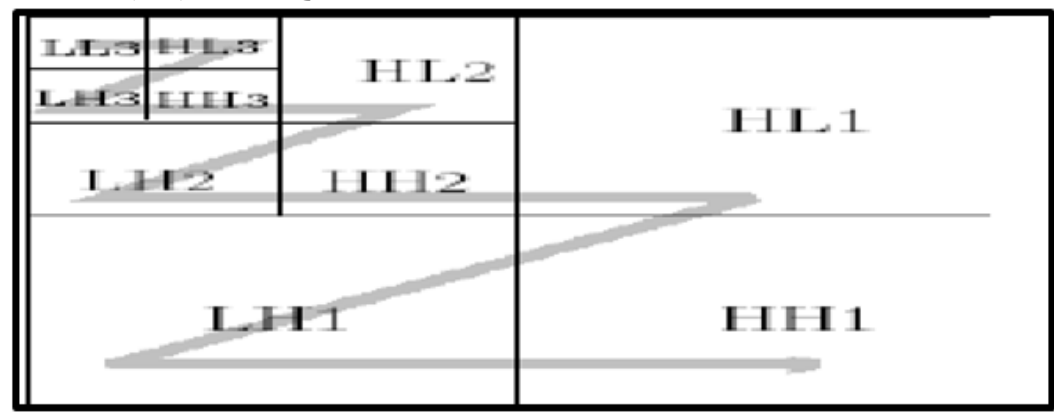

شكل(11) يوضح مفهوم الثجرة الصفرية المطمورة

(SPHIT Set Partitioning In HierarchalTree) 区 التقيم الثجري الهرمي: تعد طريقة SPHIT مطورة على EZW فبعد ان يتم اجراء عملية التحويل المويجي على الصورة, يتم ترتيب معاملات الناتجة من عملية التحويل وتدخل على ال(decoder) وال(encoder) باخذ كل معاملين متجاورين والمقارنة بينهما والنتيجة اما "1" او "0" وترسل بالوقت نفسه من الـ(decoder) سلسلة من الاصفار والواحدات الى

(encoder)

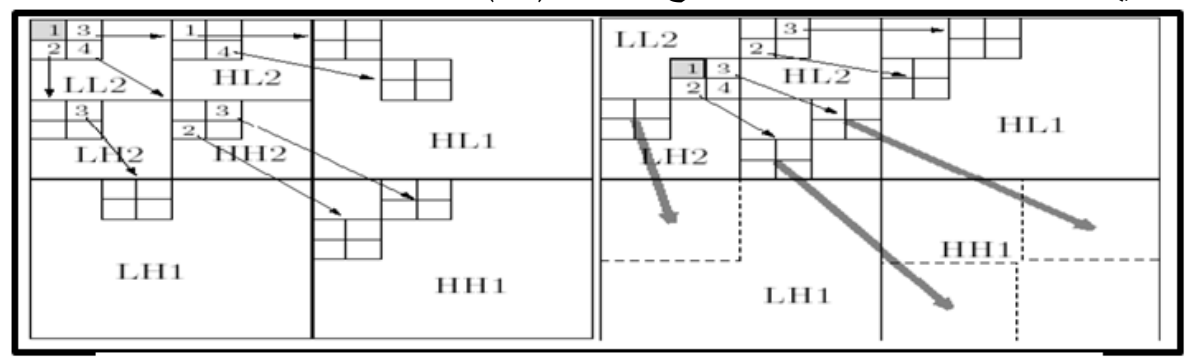

شكل(12) يوضح مفهوم التقسيم الثجري الهر مي 
وكما نلاحظ في الجدول (2) ان نسبة الكبس في الطريقة المقترحة والتي تم الاعتماد على 50 متجه مميز

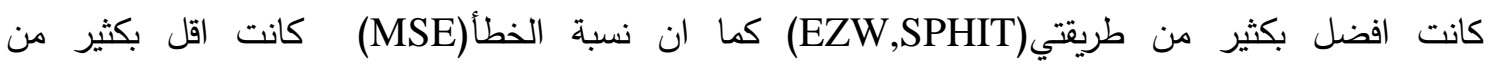
طريقتي(EZW,SPHIT) اما بالنسبة لل

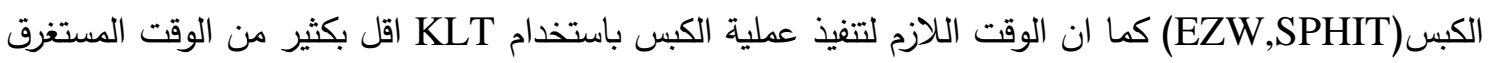

بالتفيذ باستخدام طريقتي SPHIT,EZW كمان

جدول(2) نتائج مقارنة بين طريقة KLT وطريقت EZW,SPHIT

\begin{tabular}{|c|c|c|c|c|c|}
\hline Method & MSE & $\begin{array}{c}\text { Correlation } \\
\text { Coefficient }\end{array}$ & PSNR & $\begin{array}{c}\text { Compression } \\
\text { Ratio }\end{array}$ & $\begin{array}{c}\text { execution } \\
\text { Time/second }\end{array}$ \\
\hline $\begin{array}{c}\text { Proposed } \\
\text { algorithm }\end{array}$ & 0.0037 & 0.9478 & 23.9634 & 0.1984 & $\mathbf{0 . 1 0 9 2}$ \\
\hline Ezw & 19.2811 & 0.9930 & 35.2795 & 0.9104 & $\mathbf{3 . 5 8 8 0}$ \\
\hline Sphit & 21.4635 & 0.9919 & 34.8138 & 0.6160 & $\mathbf{5 . 2 4 1 6}$ \\
\hline
\end{tabular}




$$
\text { الحيالي, ميسون خضر , 2003, "كبس صور الوثائق النصية العربية", بحث ماجستير, كلية علوم }
$$

[2] R.C. Gonzalez, R.E. Woods, 2002., "Digital Image Processing", 2nd Edition, Prentice-Hall, Jan.

[3] Y. Wang, J.Ostermann, and Y.-Q. Zhang, 2002, "Video Processing and Communications", Polytechnic University, Brooklyn, NY11201, Prentice Hall.

[4] Smith I Lindsay, February 26, 2002, "AtutorialonPrincipalComponents Analysis".

[5] Zhang Daoqiang, Chen * Songcan,2001,"Fast Image Compression using Matrix K-L Transform", Department of Computer Science and Engineering, Nanjing University of Aeronautics \& Astronautics,Nanjing 210016, P.R. China.

[6] Patricia R. Oliveira, Roseli F. Romero, 1997, "A Comparision between PCA Neural Networks and the JPEG Standard for Performing Image Compression", Authorized licensed use limited to: IEEE Xplore. Downloaded on January 19,2012 at 22:07:01 UTC from IEEE Xplore. Restrictions apply.(ivsl)

[7] Roberto Arenas Lara, Yunhong Wang,2011, "Lossless Compression On-Board Remote Sensing Satellites", Authorized licensed use limited to: IEEE Xplore. Downloaded on January 19,2012 at 22:17:48 UTC from IEEE Xplore. Restrictions apply. (ivsl)

[8] Wei Zheng1, Yan Zhang2, 2012, "A Novel Improvementto PCA for Image Classification", Authorized licensed use limited to: IEEE Xplore. Downloaded on January 19,2012 at 22:25:50 UTC from IEEE Xplore. Restrictions apply.(ivsl)

[9] Jun Ding, Zuo Zhang,2011, "A Method for Urban Traffic Data Compression Based on Wavelet-PCA", Authorized licensed use limited to: IEEExplorer. Downloaded on January 19,2012 at 22:25:58 UTC from IEEE xplore. Restrictions apply.(ivsl)

[10] Sonal, Dinesh Kumar, 2005, "A Study of Various Image Compression Techniques", Department of Computer Science \& Engineering Guru Jhambheswar University of Science and Technology, Hisar.

[11] Priti Singh, Priyanka Singh, 2001, "Design and Implementation of EZW \& SPIHT Image Coder for Virtual Images", International Journal of Computer Science and Security (IJCSS), Volume (5) : Issue (5) . 